\title{
Internal Temperature Calibration at Nanoscale on in situ Heating High Resolution Transmission Electron Microscopy
}

\author{
Yucheng Lan ${ }^{1}$, Hui Wang ${ }^{1}$, Gang Chen ${ }^{2}$ and Zhifeng Ren ${ }^{1}$ \\ 1. Department of Physics and Texas Center for Superconductivity, University of Houston, Houston, \\ Texas 77204, USA \\ 2. Department of Mechanical Engineering, Massachusetts Institute of Technology, Cambridge, \\ Massachusetts 02139, USA
}

Heating transmission electron microscopy (TEM), especial in situ heating high-resolution TEM (HRTEM) has been widely employed to in situ investigate the unique thermodynamic properties of nanomaterials, such as super-plastic properties of carbon nanotubes at high temperatures [1] and sizedependent evaporations of silver nanoparticles [2]. In most of in situ heating TEM experiments, nanoscaled temperatures were generally assumed equal to the average temperatures that were measured by traditional thermocouples built in TEM heating holders. However the two temperatures are not equal in many cases. For example, the electron beam of TEM always makes actual temperatures of observed nanoparticles deviating from average temperatures of TEM grids [3]. Therefore it is necessary to find an internal temperature calibration standard to in situ calibrate temperatures at nanoscale during in situ heating TEM experiments. In this work, an internal standard, PbTe nanocubes, was introduced to calibrate local temperatures on TEM at nanoscale and demonstrated to measure the depression melting point of silver nanoparticles.

The hydrothermally synthesized PbTe nanocubes [4] were deposited on T EM grids, pumped down, sealed in vacuumed quartz tubes, loaded into a tube furnace, and heated for 5 minutes at a temperature of $300-900{ }^{\circ} \mathrm{C}$. The temperatures of the furnace were measured by a K-type thermocouple and the temperature accuracy of the tube furnace was controlled within $\pm 1{ }^{\circ} \mathrm{C}$. Then the heated PbTe nanocubes were taken out from quartz tubes and observed on a JEOL 2010F transmission electron microscope at room temperature. Figure 1 shows TEM images of the PbTe nanocubes. Figure 1a shows $\mathrm{PbTe}$ nanocubes without heating. The morphology of the nanocubes was stable up to $300{ }^{\circ} \mathrm{C} \mathrm{(Fig.} 1 \mathrm{~b}$ ). The corners of the nanocube were rounded at $340{ }^{\circ} \mathrm{C}$ because of the melting of corners (Fig. 1c). Some tiny nanoparticles deposited around the cubes when the melted nanocubes were cooled down. When the $\mathrm{PbTe}$ nanocubes were heated at $375^{\circ} \mathrm{C}$, the edges of the nanocubes began to melt while the corners melt further (Fig. 1d). The whole nanocubes melt at $400{ }^{\circ} \mathrm{C}$ (Fig. 1e). Three critical temperatures, $340{ }^{\circ} \mathrm{C}$, $375{ }^{\circ} \mathrm{C}$ and $400{ }^{\circ} \mathrm{C}$, were determined from the morphology of the nanocubes as internal temperature calibration references.

In order to demonstrate applications of the internal temperature calibration standards, the $\mathrm{PbTe}$ nanocubes were mixed with silver nanoparticles, deposited on holey TEM grids, dried in air, and heated by a double-tilt heating TEM specimen holder in TEM column. The average temperatures heating the PbTe nanocubes were measured by a thermocouple housed in the TEM holder. In order to establish thermal equilibriums of TEM grids, the PbTe nanocubes were heated for 5 minutes at each certain heating temperature. The morphology of $\mathrm{PbTe}$ nanoparticles were in situ observed at high temperatures. Figure 2 shows in situ TEM images of a silver nanoparticle at different temperatures in vacuum. A perfect $\mathrm{PbTe}$ nanocube was located near the silver nanoparticle as an internal temperature calibration 
standard (Fig. 2a), just $500 \mathrm{~nm}$ away from the silver nanoparticle. The HRTEM image indicated that the silver nanoparticle was single crystalline at room temperature (inset in Fig. 2a). The silver nanoparticle was then in situ heated with the PbTe nanocube together. With increasing temperatures, the silver nanoparticle melted at $375{ }^{\circ} \mathrm{C}$ (Fig. 2b). HRTEM images indicated no lattice fringes of the silver nanoparticles at the temperature (inset in Fig. 2b). The morphology of the PbTe nanocube was unchanged during the heating procedure (Fig. 2a-b). Then the heating temperature was increased further and the morphology of the PbTe nanocube was in situ observed. The corner of PbTe nanocube melted first at $400{ }^{\circ} \mathrm{C}$ and the $\mathrm{PbTe}$ nanocube totally melted at $500{ }^{\circ} \mathrm{C}$ and disappeared in several minutes at 500 ${ }^{\circ} \mathrm{C}$ (Fig. 2c).

Figure $2 \mathrm{~d}$ plots the three critical melting temperatures of the PbTe naocube measured from the in situ heating TEM holder and those showed in Fig. 1. After calibration, the depression melting point of the silver nanoparticle should be $315^{\circ} \mathrm{C}, 60{ }^{\circ} \mathrm{C}$ lower than that measured from the TEM heating holder.

\section{References:}

[1] J. Y. Huang, et al., Nature 439 (2006) 281.

[2] Y. C. Lan, et al., Adv. Mater. 21 (2009), 4839.

[3] R. F. Egerton, P. Li, and M. Malac, Micron 35 (2004), 399.

[4] B. Poudel, et al., J. Nanosci. Nanotechnol. 6 (2006), 1050.

[5] The authors acknowledge funding from Defense Threat Reduction Agency under grants HDTRA110-1-0001 and HDTRA122221.
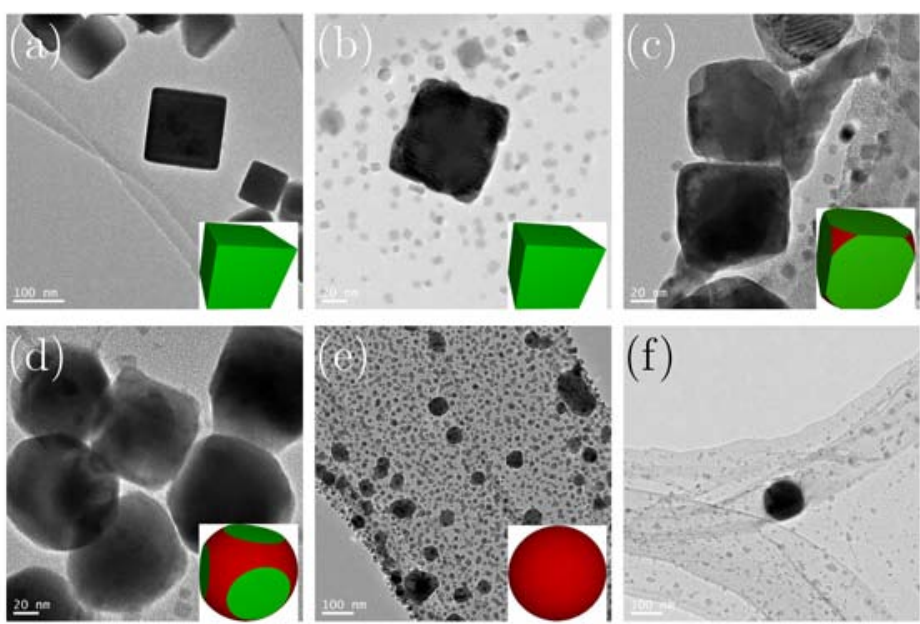

(f)

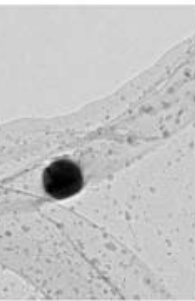

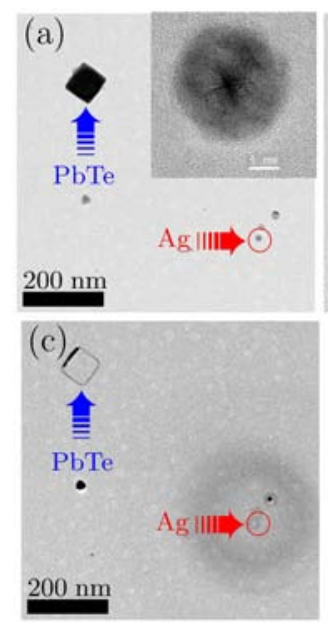

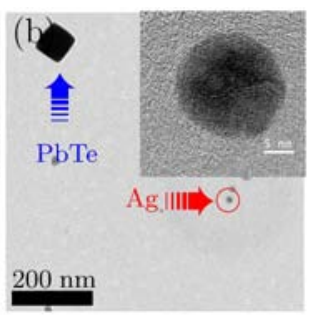

(d)

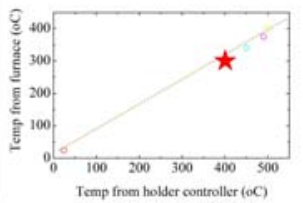

Figure 1. TEM images of heated $\mathrm{PbTe}$ nanocubes at (a) room temperature, (b) $300{ }^{\circ} \mathrm{C}$, (c) $340{ }^{\circ} \mathrm{C}$, (d) $375{ }^{\circ} \mathrm{C}$, (e) $400{ }^{\circ} \mathrm{C}$, and (f) $450{ }^{\circ} \mathrm{C}$.
Figure 2. in situ TEM images of a PbTe nanocube and a silver nanoparticle at (a) room temperature, (b) $375{ }^{\circ} \mathrm{C}$, and (c) 500 ${ }^{\circ} \mathrm{C}$. Insets: in situ HRTEM images of the silver nanoparticle. (d) in situ calibration of the melting point of the silver nanoparticle. Star: melting point of the silver nanoparticle. Square: melting points of the PbTe nanocube. --: Calibration curve. 